\title{
Objective Measurement
}

National Cancer Institute

\section{Source}

National Cancer Institute. Objective Measurement. NCI Thesaurus. Code C142618.

Ayn measurement that is not subject to observer assessment, such as those registered by an instrument. 